\title{
The atom bond connectivity index of some trees and bicyclic graphs
}

\begin{abstract}
The atom bond connectivity $(\mathrm{ABC})$ index is one of the recently most investigated degreebased molecular structure descriptors that have applications in chemistry. For a graph $\mathrm{G}$, the $A B C$ index is defined as $A B C(G)=X u v \in E(G) a ̃ d v+d u i ̄ 2 / d v \cdot d u$, where $d u$ denotes the degree of a vertex $u$ in $G$. In this paper, we obtain the general formula for $A B C$ index of some special, chemical trees, and bicyclic graphs.
\end{abstract}

Keyword: The atom bond connectivity $(\mathrm{ABC})$ index; Bicyclic graphs; Molecular graphs; Chemical tree 